\title{
Dynamics of Dispersive Wave Generation in Gas-Filled Photonic Crystal Fiber with the Normal Dispersion
}

\author{
Zhixiang Deng ${ }^{1}$ and Meng Zhang ${ }^{2}$ \\ ${ }^{1}$ School of Electrical Engineering, University of South China, Hengyang 421001, China \\ ${ }^{2}$ Oriental Science and Technology College, Hunan Agricultural University, Changsha 410082, China \\ Correspondence should be addressed to Meng Zhang; zhangmeng024678@163.com
}

Received 18 May 2017; Accepted 1 August 2017; Published 30 August 2017

Academic Editor: Xiaofeng Zhou

Copyright ( 2017 Zhixiang Deng and Meng Zhang. This is an open access article distributed under the Creative Commons Attribution License, which permits unrestricted use, distribution, and reproduction in any medium, provided the original work is properly cited.

\begin{abstract}
The absence of Raman and unique pressure-tunable dispersion is the characteristic feature of gas-filled photonic crystal fiber (PCF), and its zero dispersion points can be extended to the near-infrared by increasing gas pressure. The generation of dispersive wave (DW) in the normal group velocity dispersion (GVD) region of PCF is investigated. It is demonstrated that considering the selfsteepening (SS) and introducing the chirp of the initial input pulse are two suitable means to control the DW generation. The SS enhances the relative average intensity of blue-shift DW while weakening that of red-shift DW. The required propagation distance of DW emission is markedly varied by introducing the frequency chirp. Manipulating DW generation in gas-filled PCF by the combined effects of either SS or chirp and three-order dispersion (TOD) provides a method for a concentrated transfer of energy into the targeted wavelengths.
\end{abstract}

\section{Introduction}

The high design flexibility of photonic crystal fibers has attracted the attention of many researchers in recent decade [1]. Kagomé-lattice hollow-core PCF reported in 2002 represents a milestone in the development of microstructured fibers [2]. It demonstrates numerous additional significance properties by filling its hollow core with gas [3]. The nonlinearity and the GVD can be remarkably controlled by adjusting gas pressure or replacing of gas species $[3,4]$. The Raman-related effect disappears when the hollow core is filled with high pressure monatomic gases such as Ar, also the zero GVD point of fiber can be artificially adjusted from the ultraviolet to the near-infrared [4]. These features highly increase the versatility of hollow-core PCF, which make it an ideal platform to investigate different nonlinear optical effects.

As we all know, optical spectrum broadening and broadband frequency conversion are inherent features for nonlinear optical processes [5]. Dispersive wave (DW), also called nonsolitonic radiation or Cherenkov radiation [6], is particularly important for supercontinuum generation, broadband light sources, and broadband frequency conversion in fiber optics, and manipulating DW generation is a technique with a concentrated transfer of energy into a narrow spectral band. The generation of DW by intense optical pulses propagating, in particular, in photonic crystal fibers has been extensively studied in the past 30 years [6-9]. However, it has been originally presented at the background of the propagation of higher-order soliton. In that situation, higher-order soliton was propagated in the anomalous GVD regime which is generally perturbed by the third-and higher-order dispersion [10-13]. In our previous work, the roles of the self-steepening (SS) effect in the generation and controlling of DW in metamaterials are disclosed [14], and in photonic crystal fiber, the manipulation of DW by the frequency chirped is unfolded $[15,16]$.

In the time-domain, DW emission is described as the resonant amplification of a linear wave propagating with the same phase velocity as that of the soliton [17]. Recently, in the frequency-domain, phase-matched cascaded four-wave mixing (CFWM) is identified as the nonlinear origins of the DW generation process [18-20]. It was demonstrated that the DW emission is no longer thought to be the exclusive 
of solitons because the dispersive wave can be emitted even when pumping in the normal dispersion regime in the presence of a zero GVD wavelength [21]. In this regime, the physical origin of DW emission, which is perturbed by high-order dispersion, is intimately related to the dispersive shock waves resulting from the nonlinearity overbalancing a weak second-order dispersion [22]. The expression of the detuned frequency of dispersive waves can be accurately determined by the phase-matching selection rules, which involve the velocity of the dispersive shock waves due to emerging from a gradient catastrophe [22-24]. The roles of high-order dispersions in the generation and controlling of DW are also exposed [24-26]. However, to the best of our knowledge, so far the effect of the self-steepening and initial frequency chirp on DW generation with pumping in normal dispersion regime has not been discussed yet.

In the present paper, we demonstrate that the controllable generation of DW with pumping in the normal GVD dispersion can be realized by two means: either considering the SS effect of fiber or introducing the frequency chirp of the initial input pulse. The paper is organized as follows. In the second section, the defocusing nonlinear Schrödinger equation (dNLSE) for ultrashort pulse propagation in gasfilled photonic crystal fibers with TOD and SS effect is introduced. In the third section, we discuss the controllable DW generation and reveal the roles of the SS effect in the red-shifted and blue-shifted DW generation. In the fourth section, the impact on the DW generation on the basis of the different frequency chirp of the initial input pulse is investigated. Finally, we summarize our results.

\section{Numerical Model}

To technically elucidate the mechanism of resonant dispersive wave emission, our numerical model is based on the following normalized form of the defocusing nonlinear Schrödinger equation with the Raman term removed [27]:

$$
\frac{\partial U}{\partial \xi}+\frac{i}{2} \frac{\partial^{2} U}{\partial \tau^{2}}-\frac{\delta_{3}}{6} \frac{\partial^{3} U}{\partial \tau^{3}}=i N^{2}\left(1+i s \frac{\partial}{\partial \tau}\right)\left(|U|^{2} U\right) .
$$

Note that the Raman scattering effect is absent in the noble gas-filled PCF such as Ar considered here. We have introduced the normalized variables $\tau=\left(t-z \cdot v_{g}^{-1}\right) / T_{0}, \xi=$ $z / L_{d_{2}}$, and $U=A / \sqrt{P_{0}}$, where $T_{0}$ is the duration of the launched pulse, $P_{0}$ is the power of the input field, $v_{g}^{-1}$ is the group velocity at the central frequency $\omega_{0}$, and $L_{d_{m}}=$ $T_{0}^{m} / \beta_{m}$ is the $m$ th-order dispersion length and $L_{n l}=\left(\gamma P_{0}\right)^{-1}$ is the nonlinear length. Note that the defocusing feature arises from the assumption of the normal group velocity dispersion and nonlinear coefficient $\gamma>0 . N^{2}=L_{d_{2}} / L_{n l}$ and $\delta_{3}=L_{d_{2}} / L_{d_{3}}$ represent the normalized nonlinear coefficient and the normalized three-order dispersion coefficient, respectively. The nonlinear term in the right-hand side of (1) consists of the Kerr effect term $N^{2}|U|^{2} U$ and the shock derivative term $s N^{2} \partial\left(|U|^{2} U\right) / \partial \tau$, which gives rise to a frequency-dependent nonlinear coefficient. However, in our numerical simulation, the dispersion expansion can be truncated to the first correction to GVD, that is, third-order dispersion, whereas all the higher-order dispersive terms can be safely neglected.

To quantify ensemble frequency changes of the DW during propagation process, we introduce the intensity-weighted central frequency of the DW as a function of propagation distance $z[28]$ :

$$
\left\langle\omega_{\mathrm{DW}}(z)\right\rangle=\frac{\int_{\omega_{a}}^{\omega_{b}} \omega\left|A_{\mathrm{DW}}(\omega, z)\right| d \omega}{\int_{\omega_{a}}^{\omega_{b}}\left|A_{\mathrm{DW}}(\omega, z)\right| d \omega} .
$$

Dispersion effects are described by the first term on the righthand side of (1), where the range of integration (from $\omega_{a}$ to $\omega_{b}$ ) is selected to be no more than $-30 \mathrm{~dB}$ compared to the maximum intensity of the DW and $\left|A_{\mathrm{DW}}\right|^{2}$ represents a function of the spectral intensity of the DW with propagation distance.

In order to obtain more intuitive information of the intensity distribution of DW, the performance of the continuum spectral distribution is characterized by the decibel scale of the relative intensity [29]

$$
I(\omega, z)=10 \lg \left(\frac{|A(\omega, z)|^{2}}{\max |A(\omega, z)|^{2}}\right)
$$

and the relative average intensity of DW

$$
\eta(z)=10 \lg \left(\frac{\int_{\omega_{a}}^{\omega_{b}}|A(\omega, z)|^{2} d \omega}{\int_{-\infty}^{\infty}|A(\omega, z)|^{2} d \omega}\right) .
$$

The decibel scale used here permits us to clearly show the low-intensity radiation. However, if the intensity spectrum is presented in the form $|A(\omega, z)|^{2} / \max |A(\omega, z)|^{2}$, its value is so small that can be submerged with the background of the strong pulse.

\section{Manipulating the Dispersive Wave Generation by Self-Steepening Effect}

It was well-known that the SS effect in gas-filled photonic crystal fiber can not be ignored in practice [4]. The pulse propagating in the normal dispersion regime is also affected by the higher-order dispersion and nonlinear terms. Thus, the combined effects of the SS and TOD on the DW generation should be discussed.

To discuss the DW generation in the fiber, we employ the standard split step Fourier method to solve the dNLSE numerically. In the numerical simulation, the normalized input pulse $U(0, \tau)=\operatorname{sech}(\tau)$ is employed. For convenience, we only consider the DW generation under the condition of $N^{2}=200$. The reason for the selection can be that efficient generation of DWs from a pump in the normal GVD region typically requires the nonlinear length of the pump to be much shorter than the dispersion length of the pump. To gain a physical understanding of the effects, the loss of fiber is neglected. If not otherwise specified, only the normal GVD is considered. 


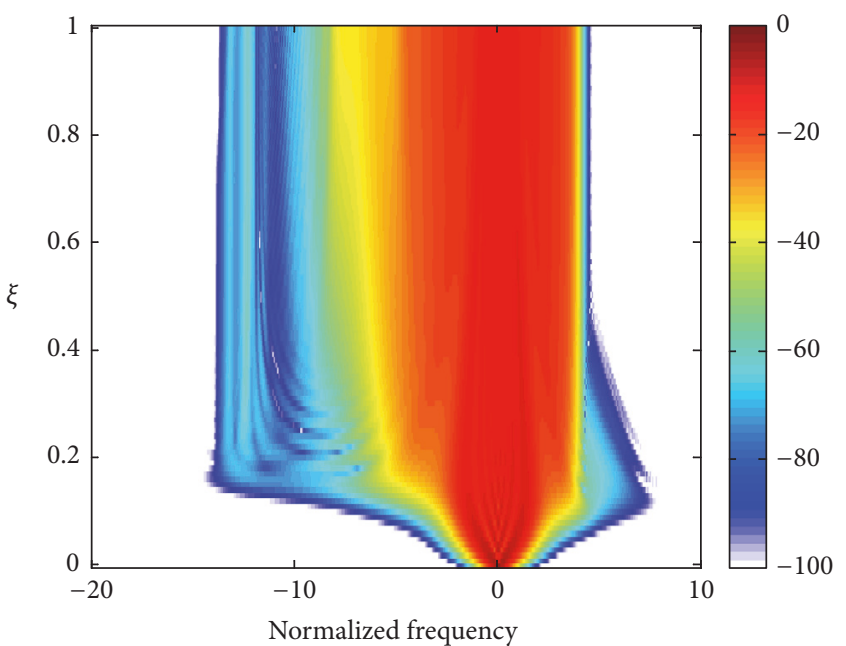

(a)

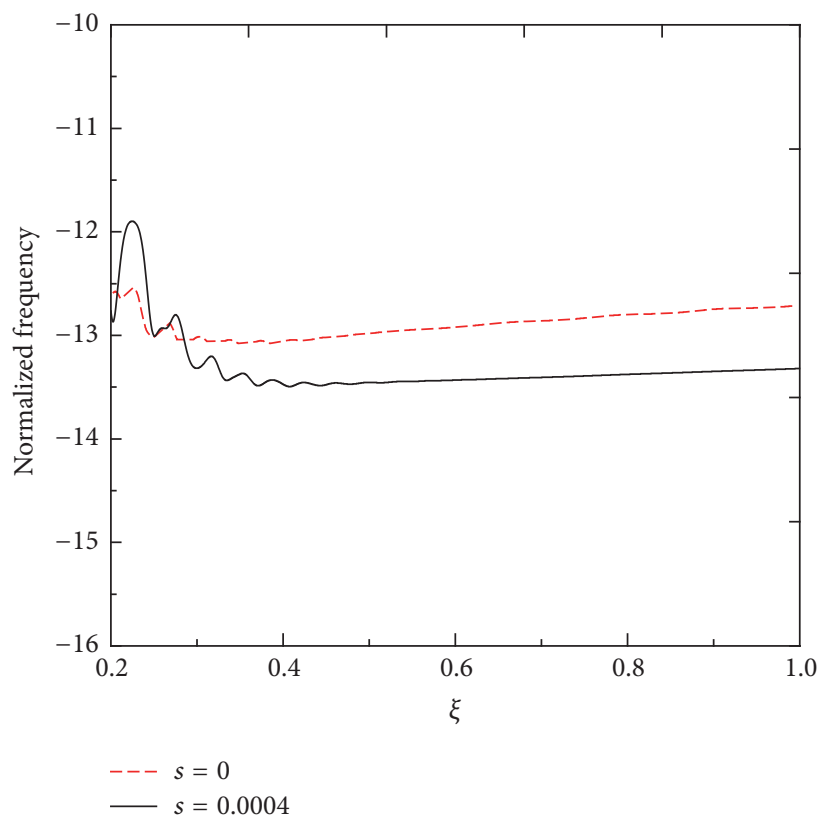

(c)

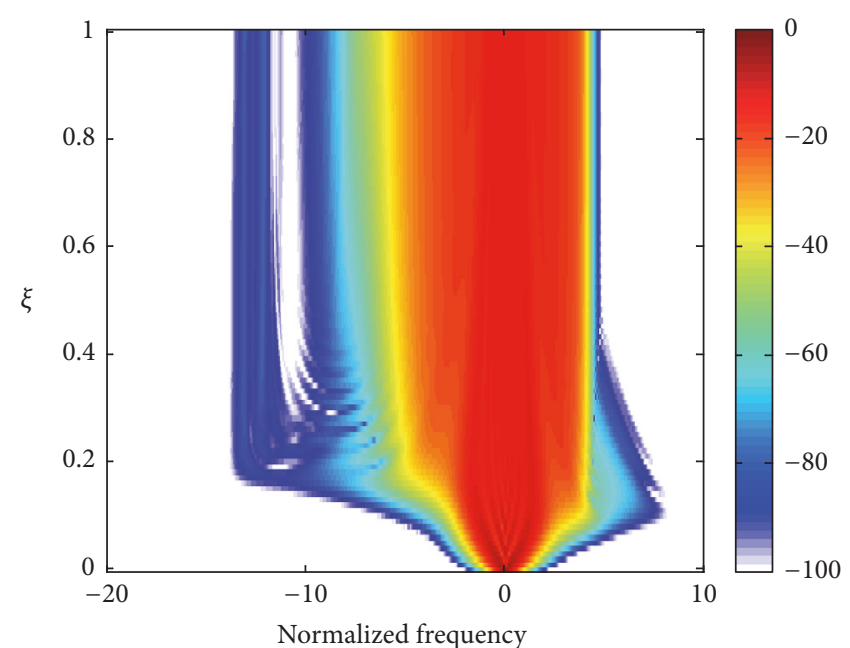

(b)

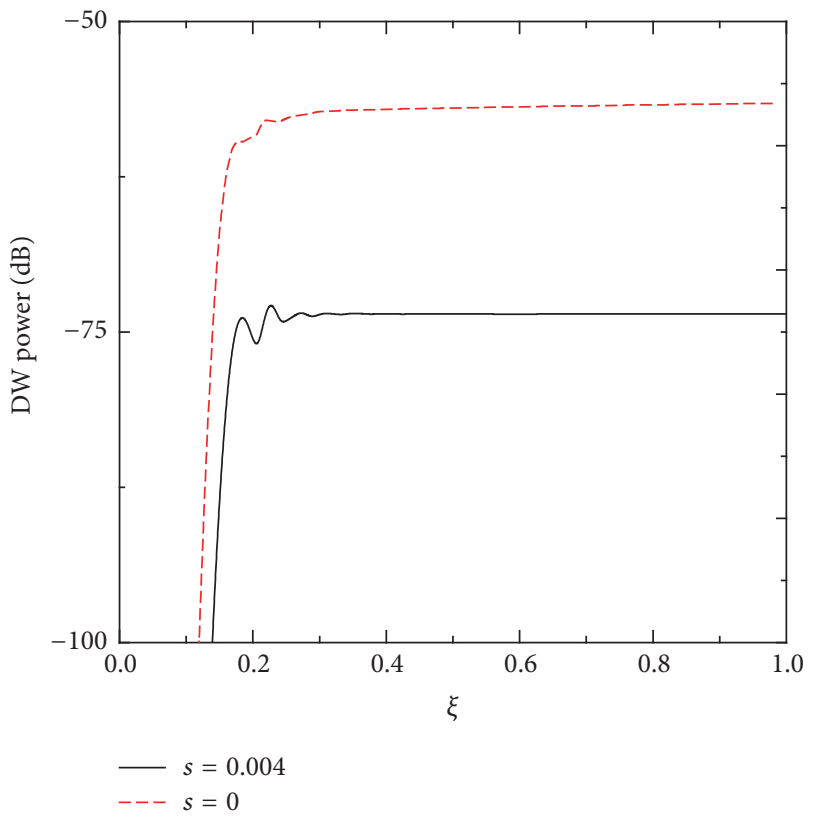

(d)

FIGURE 1: Simulate spectrum of a hyperbolic secant pulse propagating in the normal dispersion regime at the different SS coefficients, (a) $s=0$, (b) $s=0.004$, where $\delta_{3}=0.0267, N^{2}=200$. Central frequency (c) and relative average intensity (d) of the red-shift DW as a function of normalized propagation distance, where $s=0$ (red dashed curve) and $s=0.004$ (dark solid curve).

3.1. Dispersive Wave Generation for Positive Dispersion Slope. In the normal GVD of gas-filled PCF, DW emitted by dispersive chock waves owing to the positive dispersion slope (i.e., the positive TOD) will be frequency downshifted with respect to the pump. Therefore, it is called red-shift DW.

Figure 1 shows how the self-steepening effect influences the red-shift DW generation; here the positive TOD $\left(\delta_{3}=\right.$ 0.0267 ) is considered. In Figures $1(\mathrm{a})$ and $1(\mathrm{~b})$, we plot the spectral evolution of the pulse as a function of normalized propagation distance when the self-steepening effect is switched off (i.e., set $s=0$ ) and included (i.e., set $s=0.004$ ), respectively. Obviously, in the initial stages of propagation, the spectrum of the pump pulse displays strong and symmetrical broadening due to self-phase-modulation-induced pulse compresses in the presence of weak normal dispersion, but as the spectral tail of the broadened pulse overlaps with the phase-matched frequency, the occurrence of resonant energy transfer process can be seen. As the propagating distance increases, the DW emitted by dispersive shock waves begins to emerge in the red-shifted band. As can be seen by comparing Figure 1(a) with Figure 1(b), the SS effect is important for red-shift DW generation. For the two cases that the SS effect is excluded/included, the evolutions of the output spectra have the following characteristics: to begin with, the pulse spectra become narrow. When the SS effect is included, the spectrum narrowing is obvious. Therefore, this is a disadvantage to the supercontinuum generation. Secondly, the central frequency of the red-shift DW moves 


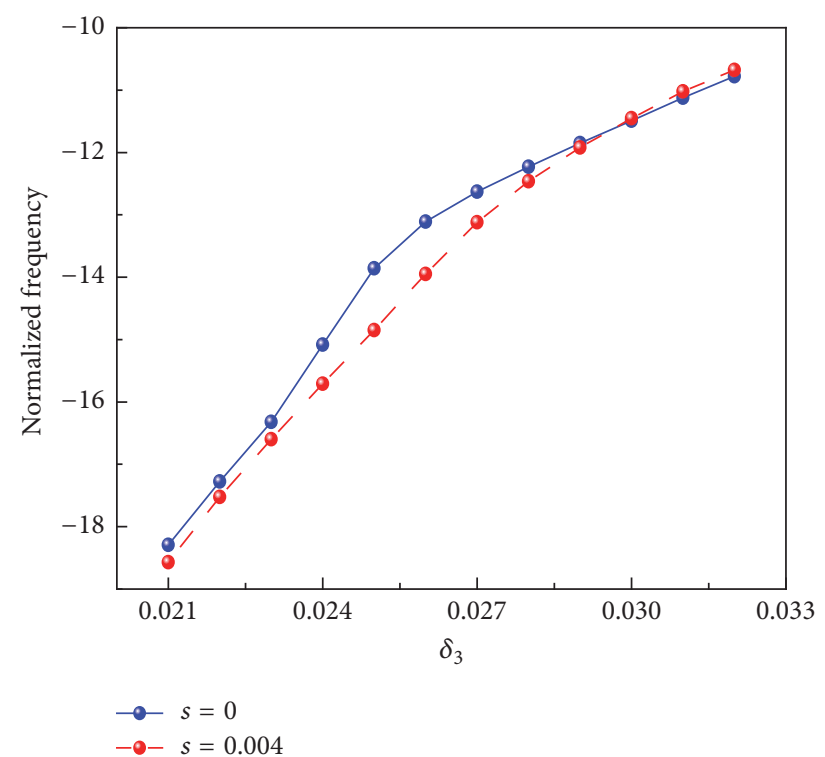

(a)

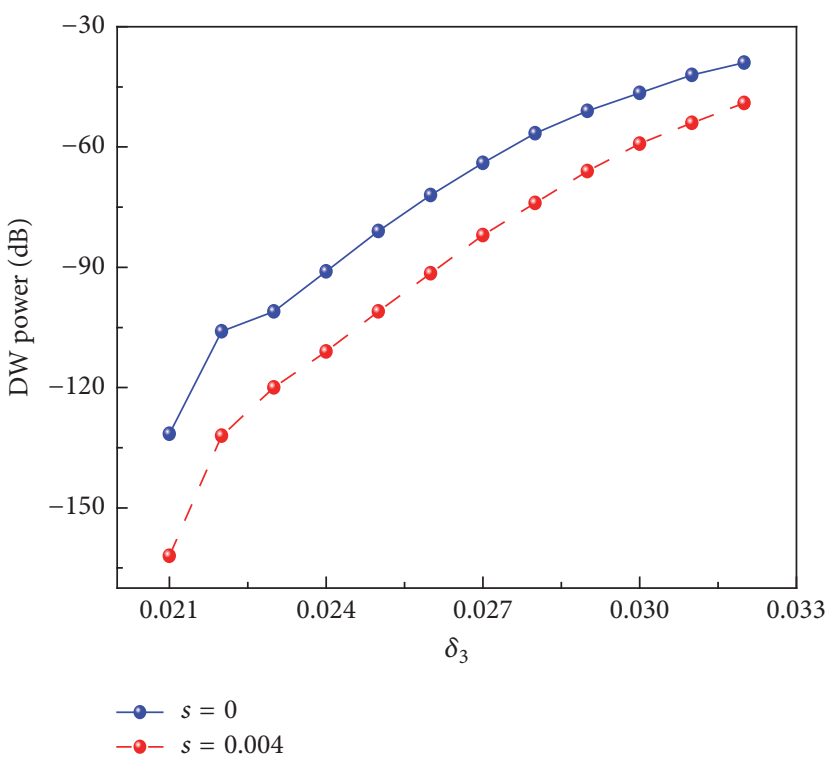

(b)

FIGURE 2: Central frequency (a) and relative average intensity (b) of the red-shift DW plotted as a function of TOD coefficient $\delta_{3}$, where $s=0$ (blue filled circles lines) and $s=0.004$ (red dashed circles lines).

away from the spectral body of the residual pump pulse. In other words, the SS effect leads to the increase of the frequency detuning $\Delta v$ from the pump. The cause of this result is that the frequency of DW is determined by the phase-matched condition associated with the nature group velocity, but SS increases the group velocity at resonant frequency eventually because it affects the group velocity in an intensity dependent fashion [30]. As can be seen from Figure $1(\mathrm{c})$, the shifting is not remarkable, which indicates that the influences of the SS effect on the frequency shifts of red-shift DW are less important than TOD. Finally, one conspicuous observation is that the relative average intensity of red-shift DW is weakened. The spectral intensity of the DW is related to the strength of the pump spectrum at the phasematched frequency $[31,32]$. Since the SS effect asymmetrically weakens the spectrum toward the red, the efficiency of DW generation dropped. As shown in Figure 1(d), the relative average intensity of red-shift DW is $-56 \mathrm{~dB}$ when the SS effect set $s=0$ and drops to $-75 \mathrm{~dB}$ when the SS effect set $s=0.004$.

To fully understand the impact of the SS effect on the red-shift DW generation, we will consider the manipulatable generation of red-shift DW by the self-steepening effect switched off or included under the different positive TOD coefficient. Next, we discuss the weighted central frequency and relative average intensity as the function of the TOD coefficient, as shown in Figure 2. We see that for the red-shift DW the frequency detuning $\Delta v$ from the pump decreases and the average intensity enhances as $\delta_{3}$ increases. In Figure 2, it also shows the influence of SS coefficient on DWs. For $\delta_{3}<$ 0.024 or $\delta_{3}>0.028$, the influence of the SS coefficient on the frequency detuning can be neglected; however, in the range $0.024<\delta_{3}<0.028$, the influence of the SS coefficient on the frequency detuning $\Delta v$ is significant. The average intensity of DWs is influenced by the SS effect distinctly; however, in contrast to the case of without SS effect, the average intensity of DWs reduces to over $20 \mathrm{~dB}$ when the SS effect is included. Based on these analysis results, we reach the conclusion that the SS effect weakens the red-shift DW generation. For instance, when $\delta_{3}=0.032$, the average intensity is about $-40 \mathrm{~dB}$ if SS effect is switched off; however, it can be weakened by the SS coefficient to $-55 \mathrm{~dB}$ at $s=0.004$.

\subsection{Dispersive Wave Generation for Negative Dispersion Slope.} The former section just considers the role of the positive TOD parameter in the DW generation. Indeed, we can also obtain the negative value of TOD parameter by varying either the pressure or temperature of the filling gas in the photonic crystal fibers. The case is that the dispersive wave appears to be located at higher frequencies compared with our pump pulse; therefore, it is the so-called the blue-shift DW. Next, we turn to the discussion about the role of the negative TOD combined with SS effect in the blue-shift DW generation. For $\delta_{3}=-0.0267$, Figures 3(a) and 3(b) show the contour of the injected pulse when the SS effect is switched off and included, respectively. In stark contrast to the result of the positive TOD, the evolutions of the output spectra based on whether the SS effect is included or not have the distinct feature as follows: firstly, the pulse spectra become narrow. When the SS effect is included, the spectrum broadening is obvious. Therefore, this is favorable to the supercontinuum generation. Next, the central frequency of blue-shift DW moves toward the spectral body of the residual pump pulse. In other words, for blue-shift DW, the frequency detuning $\Delta v$ from the pump pulse is decreased. Reasonable explanation is that the SS effect alters the group velocity associated with the expression of the phase-matching condition. As can be seen from Figure $3(\mathrm{c})$, at $\xi=1$, the average location of blue-shift DW when the SS effect is ignored is 0.3 above that 


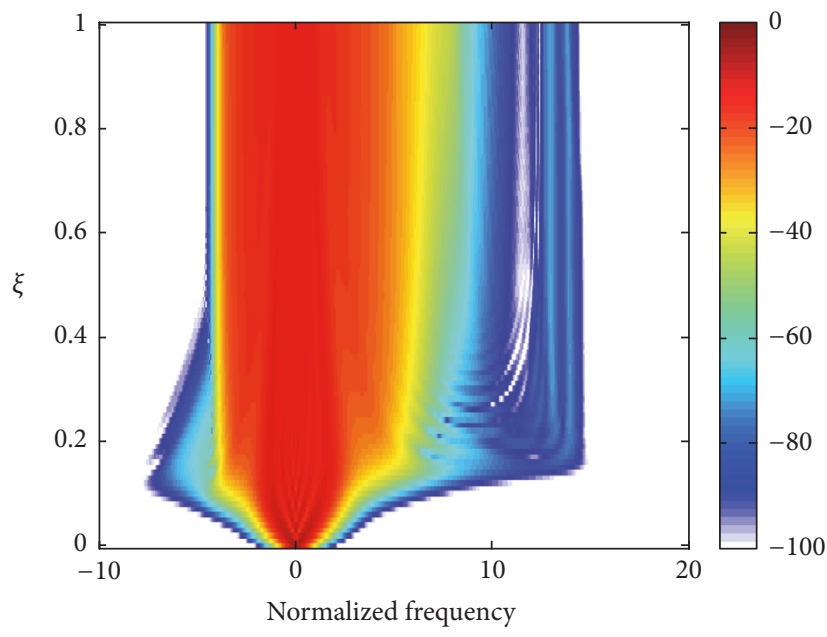

(a)

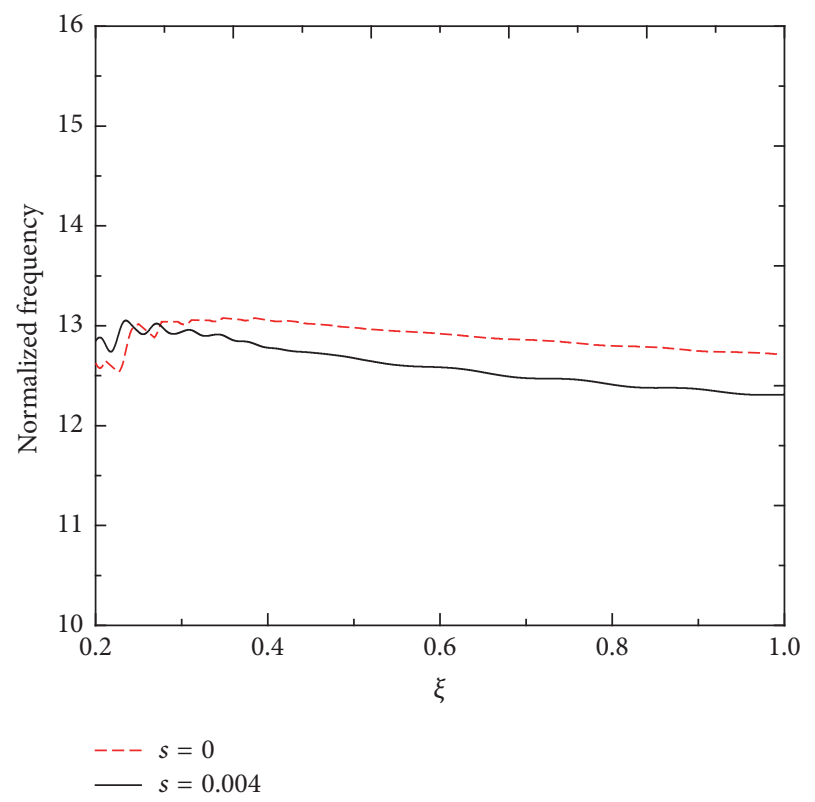

(c)

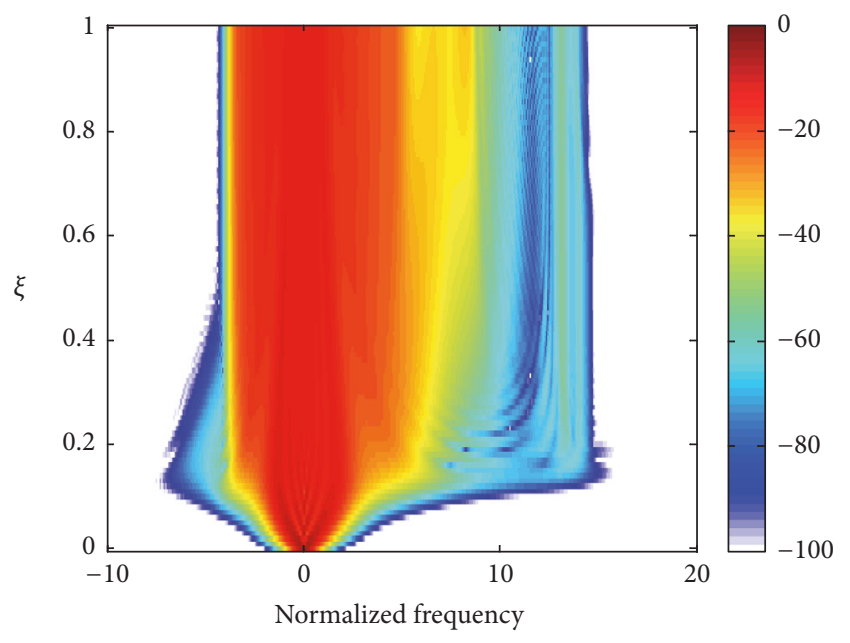

(b)

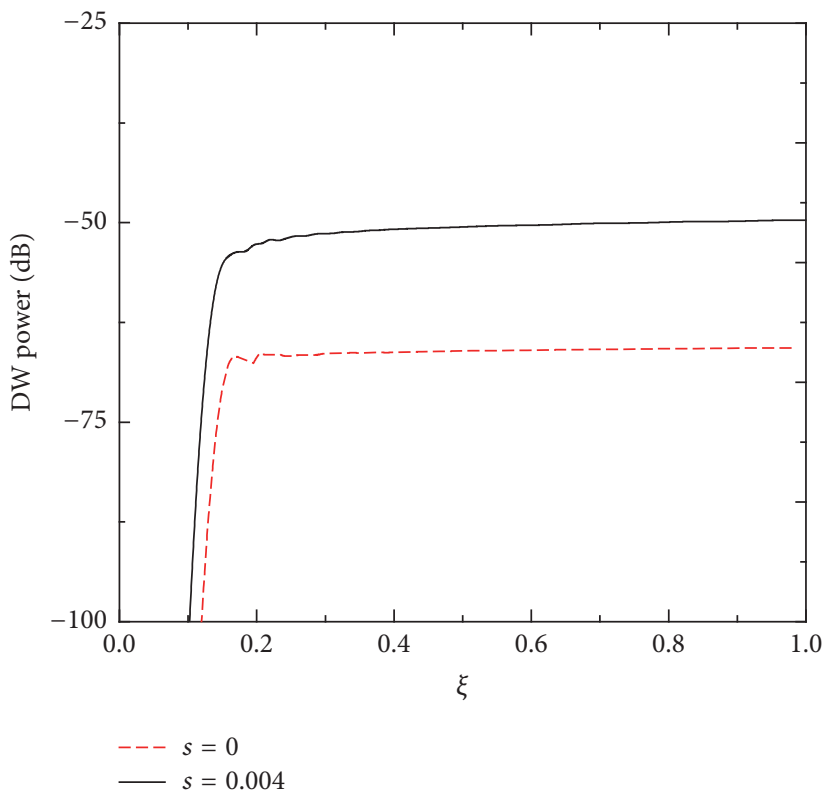

(d)

FIGURE 3: Simulate spectrum of a hyperbolic secant pulse propagating in the normal dispersion regime at the different SS coefficients, (a) $s=0$ and (b) $s=0.004$, where $\delta_{3}=-0.0267$ and $N^{2}=200$. Central frequency (c) and the relative average intensity (d) of the blue-shift DW as a function of normalized propagation distance, where $s=0$ (red dashed curve) and $s=0.004$ (dark solid curve).

of blue-shift DW when it is taken into account. Ultimately, the relative average intensity of blue-shift DW is enhanced. Since the effect of SS asymmetrically enhances the spectrum toward the blue, it is improving the efficiency of blue-shift DW generation. As shown in Figure 3(d), the relative average intensity of blue-shift DW is $-67 \mathrm{~dB}$ when the SS effect is switched off, while it increases to $-49 \mathrm{~dB}$ when it is included.

To further get a thorough understanding of the influence of the SS effect on the blue-shift DW generation, Figure 4 indicates, on the basis of the two cases of the SS effect are included or not, the frequency detuning and relative average intensity of the blue-shift DW plotted as a function of negative TOD coefficient. In both cases, as can be seen from Figure 4(a), the blue-shift DW shifts to lower frequency with the increasing $\left|\delta_{3}\right|$. However, note that the frequency detuning $\Delta v$ of the blue-shift DW in the presence of the SS effect is lower than that of the DW neglecting the SS effect, especially when $\left|\delta_{3}\right|$ is placed in the range $0.022<$ $\left|\delta_{3}\right|<0.027$. As shown in Figure 4(b), the relative average intensity of DWs enhanced as TOD coefficient $\left|\delta_{3}\right|$ increases; nonetheless, it is noted that the amount of relative average intensity of the DW when the SS effect is neglected is about $20 \mathrm{~dB}$ below that of the DW when it is included. These results indicate that the relative average intensity of blue-shift DW is enhanced, rather weakened, and the average position of blue-shift DW can be shifted slightly to the spectral body of the residual pump pulse via considering the SS effect. 


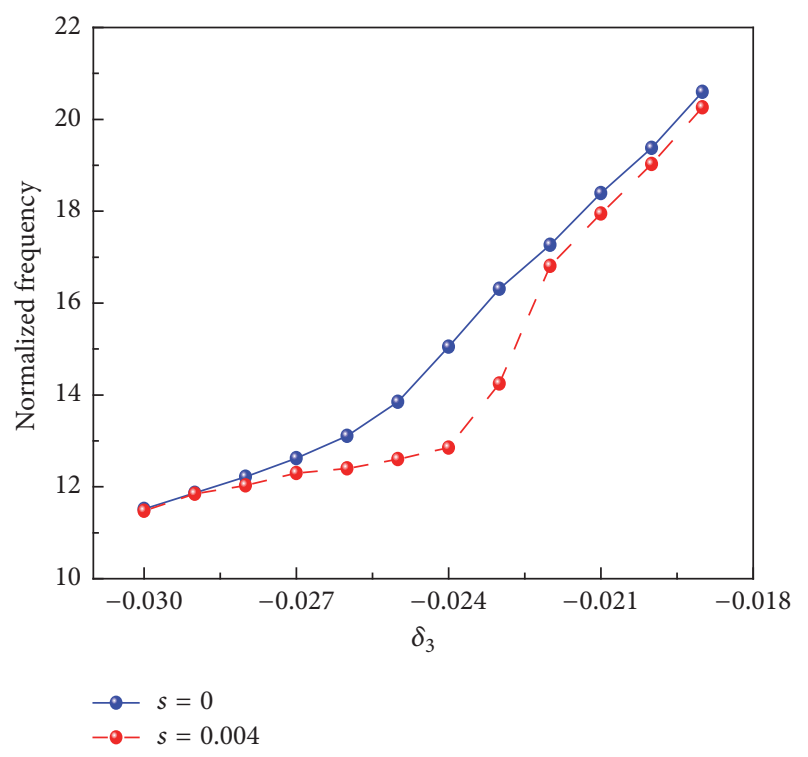

(a)

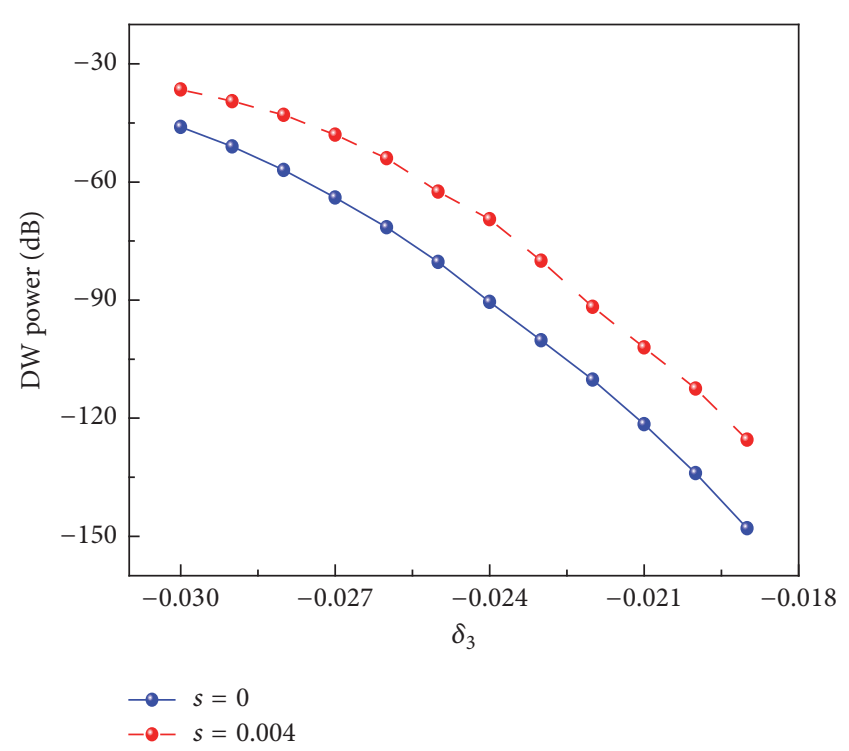

(b)

FIGURE 4: Central frequency (a) and relative average intensity (b) of the blue-shift DW plotted as a function of TOD coefficient $\delta_{3}$, where $s=0$ (blue filled circles lines) and $s=0.004$ (red dashed circles lines).

\section{Manipulating the Dispersive Wave Generation by Frequency Chirp}

From the realistic perspective, pulses emitted from laser sources are often chirped [33]. Therefore, apart from the self-steepening effect of dispersive waves emission discussed above, good understanding of the frequency chirp influence on dispersive waves emission is of great importance. In the section, we consider in detail the effect of initial frequency chirp on DW generation. In order to understand the pulse evolution, that is, the spectral expansion and DW generation, under the influence of pulse chirp we numerically simulated the pulse amplitude propagation using a split step Fourier simulation tool, as well. In order to highlight the role of frequency chirp on the DW generation, the SS effect in the next discussion is switched off. In our simulation, the initial incident pulse is assumed to have a normalized form of hyperbolic secant field profile:

$$
U(0, \tau)=\operatorname{sech}(\tau) \exp \left(-\frac{i C \tau^{2}}{2}\right)
$$

where $C$ is the parameter representing the initial linear frequency chirp. We started our simulations to verify this qualitative behavior.

4.1. Dispersive Wave Generation for Positive Dispersion Slope. For the sake of convenience, we supposed that the positive TOD coefficient is fixed at $\delta_{3}=0.0267$, that is, set $\delta_{3}=$ 0.0267 . To better understand the effects of initial chirps, a series of spectral evolutions for different input chirps is displayed in Figure 5. Comparing Figures 5(a)-5(c), it is observed that the frequency chirp is vital to the DW generation. With the frequency chirp changing from negative to positive, the evolutions of the output spectra have the following characteristics: firstly, the distance at which the spectral broaden to its maximum value is modified. In other words, the propagation distance which DW generation required is changed. If no chirp is applied, the initial pulse spectrum can be seen to approach a maximum spectrum width within the first $0.15 L_{d_{2}}$ of propagation distance (see Figure 5(b)). If a negative input chirp is applied, the needed distance at which the DW generated is earlier compare to that of unchirped pulse [see black dash line in Figure 5(a)]. More interesting, Figure 5(c) shows that the initial pulse spectrum approach a maximum point is deferred when a positive input chirp is applied. The reason for this is that, for positive chirp coefficient, the pulse duration increases with distance due to $\beta_{2} C>0$. The decrease in intensity then lessens the nonlinear effects, leading to slow the rate of bandwidth broadening, whereas for a negative chirp coefficient, the pulse duration first decreases until it reaches minimum value at a distance due to $\beta_{2} C<0$. Such, the increase in intensity then enhances the nonlinear effects further, resulting in speed up the rate of bandwidth broadening. This consideration explains why the positive chirped pulse emits the DW later, or the negative chirped pulse emits the DW earlier compare to the case of unchirped pulse.

Secondly, the frequency centre of DW during the propagation is markedly changed. As can be seen from Figure 6(a), in the initial stage of the DW generation, the frequency detuning between the DW and the pump pulse gradually decrease when the parameter values $C$ of the initial input chirp increased from -2 to 2 (note that we only draw the evolution of centre frequency of DW with propagation distance beyond $0.2 L_{d_{2}}$ because of no DW generation in the earlier propagation). With the further increase of the propagation distance, the central frequency of DW becomes 


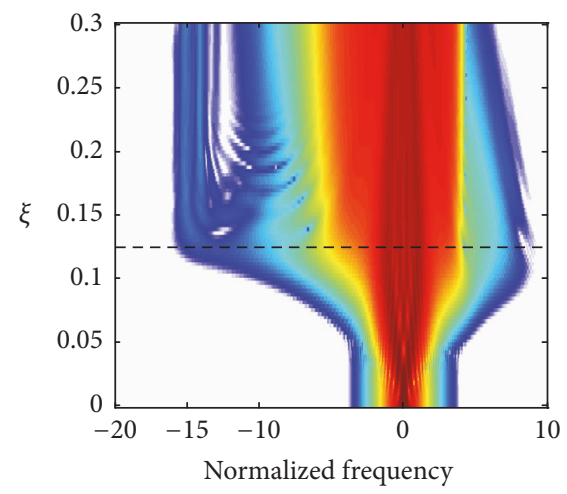

(a)

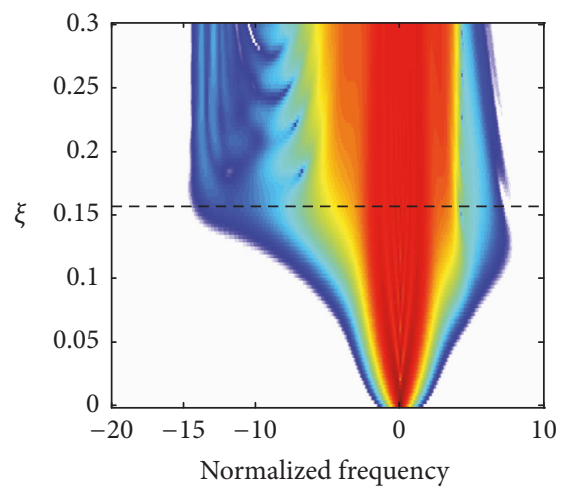

(b)

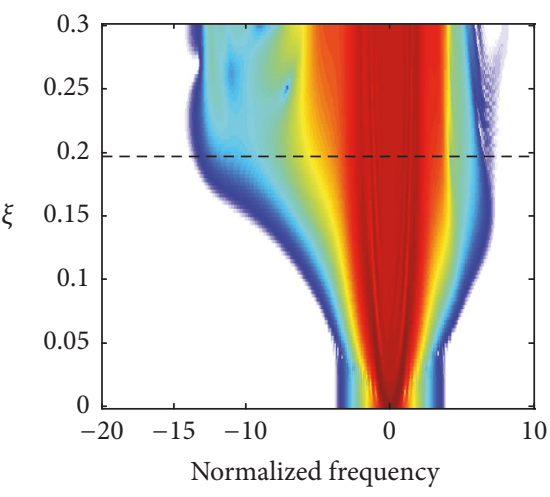

(c)

Figure 5: Pulse spectral evolution during propagation for different input pulse chirps, (a) $C=-2$, (b) $C=0$, and (c) $C=2$ as the sign of TOD is positive. The black horizontal dash line indicates the position at which the maximum spectral broadening occurs, that is, the DW generated.

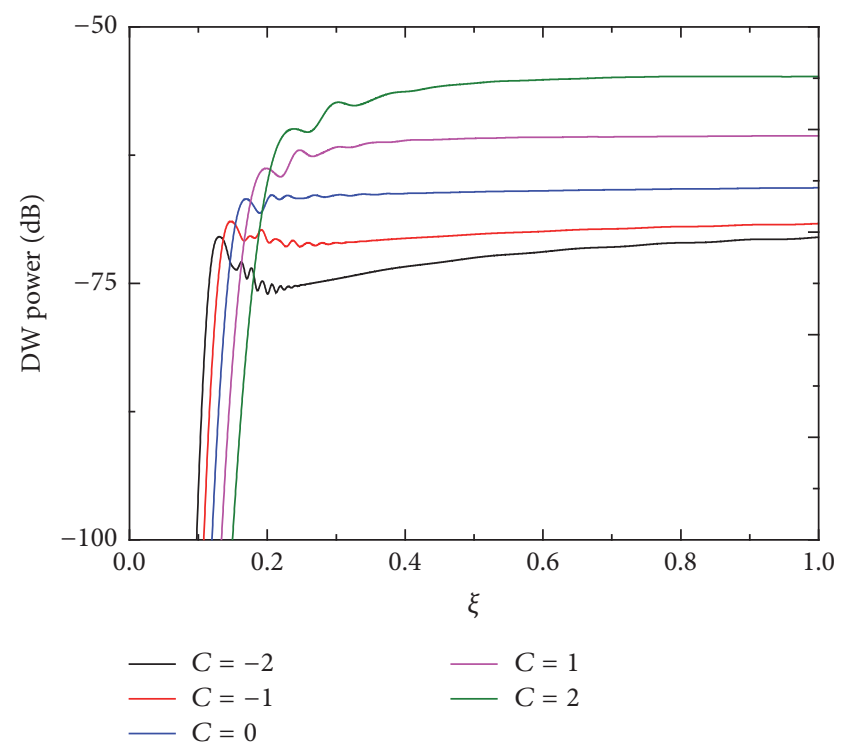

(a)

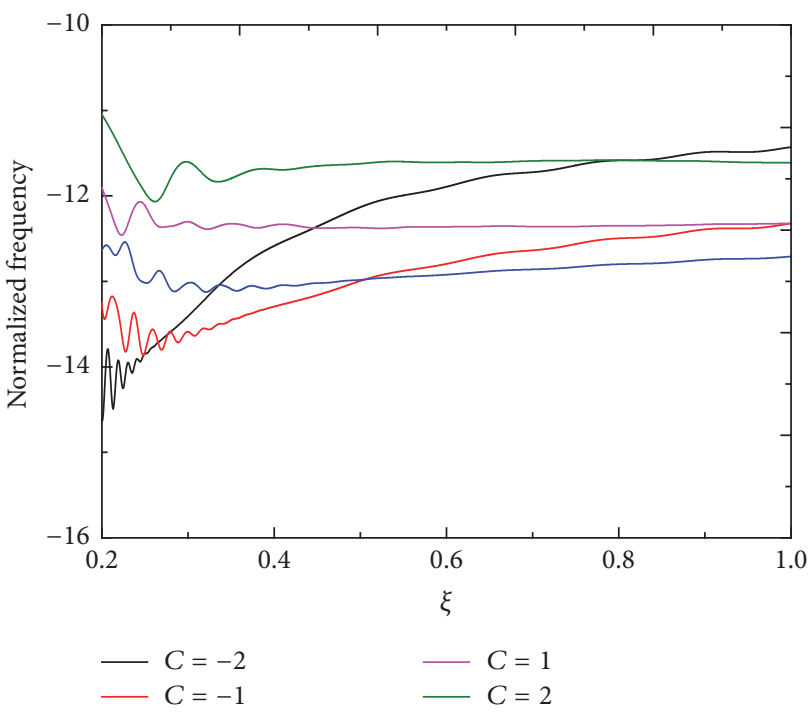

(b)

FIGURE 6: Evolution of the red-shift DW of relative average power (a) and central frequency (b) along the propagation distance, with different chirp but the sign of TOD is positive: dark solid curves $C=-2$; red solid curves $C=-1$; blue solid curves $C=0$; magenta solid curves $C=1$; olive solid curves $C=2$.

saturated quickly when a positive input chirp is applied; however, for a negative input chirp, the central frequency of DW can be seen to shift gradually toward the spectral body of residual pump, and eventually, the frequency detuning $\Delta v$ from the pump pulse will be less than the case in the absence of the initial frequency chirp.

Finally, the relative average intensity of DW can be either enhanced or reduced. The dependence of relative average power on propagation distance is displayed in Figure 6(b) for a set of input pulse chirp parameters. From the figure, it is apparent that, in contrast to that of the unchirped input pulse, the positive chirp enhance the average intensity of the DW while the negative chirp weakens that of the DW. However, regardless of the sign of the initial input chirps, the average intensity of DW becomes saturated rapidly along fiber propagation.
4.2. Dispersive Wave Generation for Negative Dispersion Slope. For completeness, in the following sections we considered the case of negative dispersion slope, that is, negative TOD. In order to better compare with the case of positive TOD discussed above, we will continue to the fixed TOD coefficient and set $\delta_{3}=-0.0267$. The simulated optical spectral evolution of the pulse with three different chirp parameters is shown in Figures $7(a)-7(\mathrm{c})$. The required distance at which the DW emitted, the central frequency of DW and the relative average intensity of the DW are obviously altered as the frequency chirp is applied. More interesting, the transformation law plotted in Figure 8 is almost the same as the case of positive dispersion slope. This indicates that the effect of frequency chirp to blue-shift DW will be the same as that of red-shift DW. This provides a means to tune the DW emission by varying the initial chirp of the input pulse. 


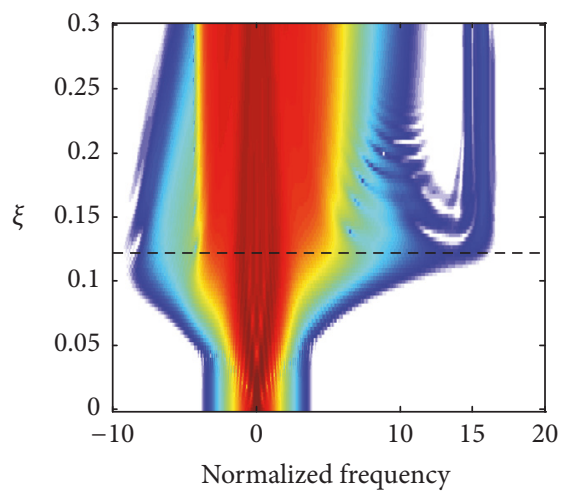

(a)

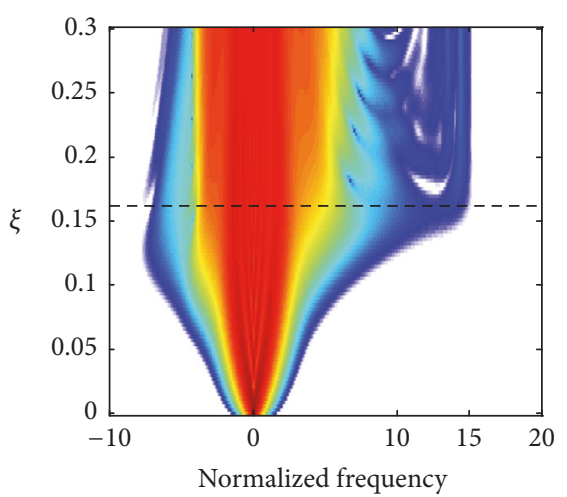

(b)

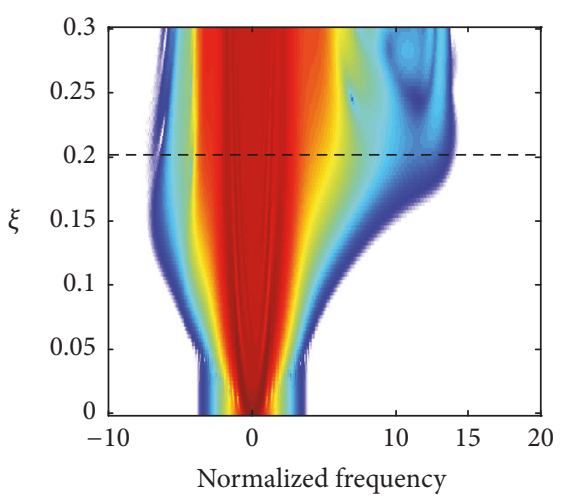

(c)

Figure 7: Density plots of spectral evolution during propagation for different input pulse chirps, (a) $C=-2$, (b) $C=0$, and (c) $C=2$ when the sign of TOD is negative. The black horizontal dash line indicates the position at which the maximum spectral broadening occurs, that is, the DW generated.

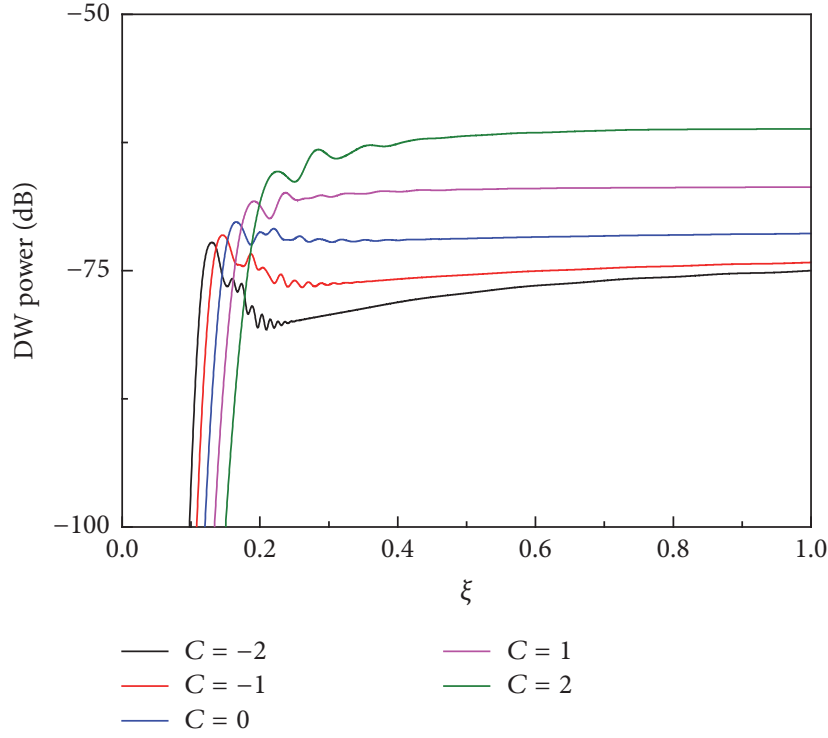

(a)

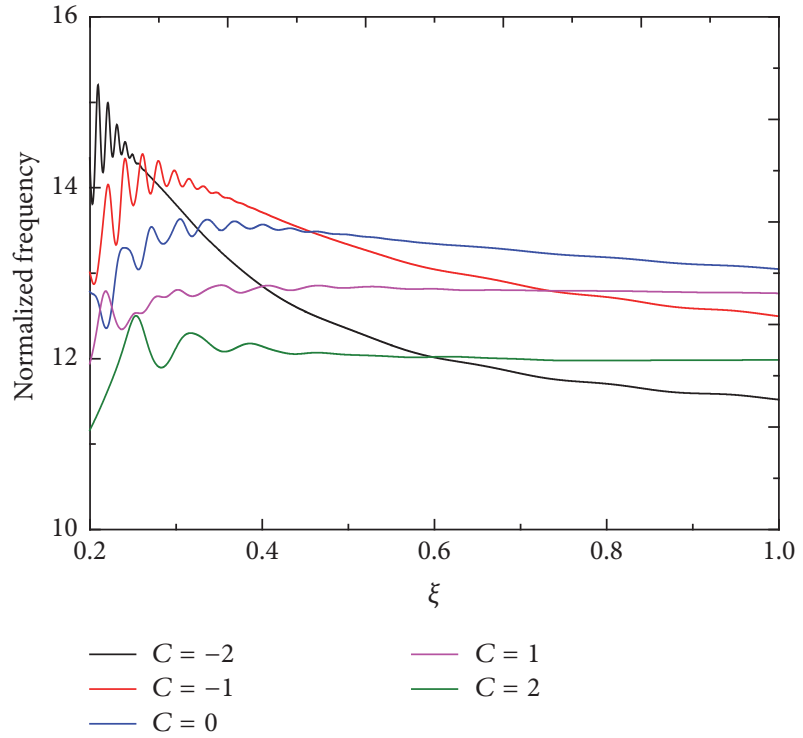

(b)

Figure 8: Evolution of the blue-shift DW of central frequency (a) and relative average intensity (b) along the propagation distance, with different chirp but the sign of TOD is negative: dark solid curves $C=-2$; red solid curves $C=-1$; blue solid curves $C=0$; magenta solid curves $C=1$; olive solid curves $C=2$.

\section{Conclusions}

In summary, we have presented numerical results describing the nonlinear propagation of the ultrashort pulse in normal dispersion region in gas-filled photonic crystal fiber. When it satisfies the phase-matching condition at the different signs of dispersion slope, it will produce red-shift or blue-shift DW relative to the input pump pulse. Our results suggest that the properties of red-shifted or blue-shifted DW can be markedly manipulated by the SS effect. For the red-shifted DW, the relative average intensity of DW decreases rapidly and the central frequency of DW downshifts slightly when the SS effect is considered. However, the results are diametrically opposite to the case of blue-shifted DW. The effect of initial chirp on the DW generation is also studied. Regardless of the red-shifted or blue-shifted DW, the negative chirp can speed up the DW generation while the positive chirp will slow it down. Meanwhile, the frequency chirp leads to marked changing of the relative average power and central frequency of DW, as well. Manipulating DW generation in gas-filled PCF by the combined effects of either SS or chirp and threeorder dispersion provides an alternative route for an efficient and concentrated transfer of energy into the desired spectral bands.

\section{Conflicts of Interest}

The authors declare that they have no conflicts of interest. 


\section{Acknowledgments}

This work was supported by the National Natural Science Foundation of China [Grant nos. 61571183, 61505122] and the Guangdong Natural Science Foundation (Grant no. 2014A030310279).

\section{References}

[1] P. S. J. Russell, "Photonic crystal fibres," Science, vol. 299, no. 5605, pp. 358-362, 2003.

[2] F. Benabid, J. C. Knight, G. Antonopoulos, and P. S. J. Russell, "Stimulated Raman scattering in hydrogen-filled hollow-core photonic crystal fiber," Science, vol. 298, no. 5592, pp. 399-402, 2002.

[3] P. S. J. Russell, P. Hölzer, W. Chang, A. Abdolvand, and J. C. Travers, "Hollow-core photonic crystal fibres for gas-based nonlinear optics," Nature Photonics, vol. 8, no. 4, pp. 278-286, 2014.

[4] J. C. Travers, W. Chang, J. Nold, N. Y. Joly, and P. S. J. Russell, "Ultrafast nonlinear optics in gas-filled hollow-core photonic crystal fibers," Journal of the Optical Society of America B: Optical Physics, vol. 28, no. 12, pp. A11-A26, 2011.

[5] J. M. Dudley, G. Genty, and S. Coen, "Supercontinuum generation in photonic crystal fiber," Reviews of Modern Physics, vol. 78, no. 4, pp. 1135-1184, 2006.

[6] N. Akhmediev and M. Karlsson, "Cherenkov radiation emitted by solitons in optical fibers," Physical Review A, vol. 51, no. 3, pp. 2602-2607, 1995.

[7] P. K. A. Wai, H. H. Chen, and Y. C. Lee, "Radiations by solitons at the zero group-dispersion wavelength of single-mode optical fibers," Physical Review A, vol. 41, no. 1, pp. 426-439, 1990.

[8] P. K. A. Wai, C. R. Menyuk, Y. C. Lee, and H. H. Chen, "Nonlinear pulse propagation in the neighborhood of the zerodispersion wavelength of monomode optical fibers," Optics Letters, vol. 11, no. 7, pp. 464-466, 1986.

[9] V. I. Karpman, "Radiation by solitons due to higher-order dispersion," Physical Review E. Statistical, Nonlinear, and Soft Matter Physics, vol. 47, no. 3, pp. 2073-2082, 1993.

[10] H. Yang, F. Han, H. Hu, W. Wang, and Q. Zeng, "Spectraltemporal analysis of dispersive wave generation in photonic crystal fibers of different dispersion slope," Journal of Modern Optics, vol. 61, no. 5, pp. 409-414, 2014.

[11] K. M. Hilligsøe, H. N. Paulsen, J. Thøgersen, S. R. Keiding, and J. J. Larsen, "Initial steps of supercontinuum generation in photonic crystal fibers," Journal of the Optical Society of America B: Optical Physics, vol. 20, no. 9, pp. 1887-1893, 2003.

[12] S. Roy, S. K. Bhadra, and G. P. Agrawal, "Perturbation of higherorder solitons by fourth-order dispersion in optical fibers," Optics Communications, vol. 282, no. 18, pp. 3798-3803, 2009.

[13] J. N. Elgin, T. Brabec, and S. M. J. Kelly, "A perturbative theory of soliton propagation in the presence of third order dispersion," Optics Communications, vol. 114, no. 3-4, pp. 321-328, 1995.

[14] S. Hill, C. E. Kuklewicz, U. Leonhardt, and F. König, "Evolution of light trapped by a soliton in a microstructured fiber," Optics Express, vol. 17, no. 16, pp. 13588-13601, 2009.

[15] X. Fu, L. Qian, S. Wen, and D. Fan, "Nonlinear chirped pulse propagation and supercontinuum generation in microstructured optical fibre," Journal of Optics A: Pure and Applied Optics, vol. 6, no. 11, pp. 1012-1016, 2004.
[16] D. Lei, H. Dong, S. Wen, and H. Yang, "Manipulating dispersive wave generation by frequency chirp in photonic crystal fibers," Journal of Lightwave Technology, vol. 27, no. 20, pp. 4501-4507, 2009.

[17] A. V. Husakou and J. Herrmann, "Supercontinuum generation of higher-order solitons by fission in photonic crystal fibers," Physical Review Letters, vol. 87, no. 20, Article ID 203901, 2001.

[18] M. Erkintalo, Y. Q. Xu, S. G. Murdoch, J. M. Dudley, and G. Genty, "Cascaded phase matching and nonlinear symmetry breaking in fiber frequency combs," Physical Review Letters, vol. 109, no. 22, Article ID 223904, 2012.

[19] K. E. Webb, M. Erkintalo, Y. Xu et al., "Nonlinear optics of fibre event horizons," Nature Communications, vol. 5, article no. 4969, 2015.

[20] K. E. Webb, M. Erkintalo, Y. Q. Xu, G. Genty, and S. G. Murdoch, "Efficiency of dispersive wave generation from a dual-frequency beat signal," Optics Letters, vol. 39, no. 20, pp. 5850-5853, 2014.

[21] K. E. Webb, Y. Q. Xu, M. Erkintalo, and S. G. Murdoch, "Generalized dispersive wave emission in nonlinear fiber optics," Optics Letters, vol. 38, no. 2, pp. 151-153, 2013.

[22] M. Conforti and S. Trillo, "Dispersive wave emission from wave breaking," Optics Letters, vol. 38, no. 19, pp. 3815-3818, 2013.

[23] J. Fatome, C. Finot, G. Millot, A. Armaroli, and S. Trillo, "Observation of optical undular bores in multiple four-wave mixing," Physical Review X, vol. 4, no. 2, Article ID 021022, 2014.

[24] M. Conforti, F. Baronio, and S. Trillo, "Resonant radiation shed by dispersive shock waves," Physical Review A - Atomic, Molecular, and Optical Physics, vol. 89, no. 1, Article ID 013807, 2014.

[25] S. Malaguti, M. Conforti, and S. Trillo, "Dispersive radiation induced by shock waves in passive resonators," Optics Letters, vol. 39, no. 19, pp. 5626-5629, 2014.

[26] S. Malaguti, G. Bellanca, and S. Trillo, "Dispersive wavebreaking in coherently driven passive cavities," Optics Letters, vol. 39, no. 8, pp. 2475-2478, 2014.

[27] N. Y. Joly, J. Nold, W. Chang et al., "Bright spatially coherent wavelength-tunable deep-UV laser source using an Ar-filled photonic crystal fiber," Physical Review Letters, vol. 106, no. 20, Article ID 203901, 2011.

[28] C. Liu, E. J. Rees, T. Laurila, S. Jian, and C. F. Kaminski, “Periodic interactions between solitons and dispersive waves during the generation of noncoherent supercontinuum radiation," Optics Express, vol. 20, no. 6, pp. 6316-6324, 2012.

[29] D. A. Korobko, O. G. Okhotnikov, D. A. Stoliarov, A. A. Sysolyatin, and I. O. Zolotovskii, "Broadband infrared continuum generation in dispersion shifted tapered fiber," Journal of the Optical Society of America B: Optical Physics, vol. 32, no. 4, pp. 692-700, 2015.

[30] G. P. Agrawal, Nonlinear Fiber Optics, Academic, New York, 4th edition, 2007.

[31] T. Marest, C. Mas Arabí, M. Conforti et al., "Emission of dispersive waves from a train of dark solitons in optical fibers," Optics Letters, vol. 41, no. 11, pp. 2454-2457, 2016.

[32] Y. Chen, G. Jiang, S. Chen et al., "Mechanically exfoliated black phosphorus as a new saturable absorber for both Q-switching and mode-locking laser operation," Optics Express, vol. 23, no. 10, pp. 12823-12833, 2015.

[33] S. Chen, L. Miao, X. Chen et al., "Few-layer topological insulator for all-optical signal processing using the nonlinear kerr effect," Advanced Optical Materials, vol. 3, no. 3, pp. 1769-1778, 2015. 

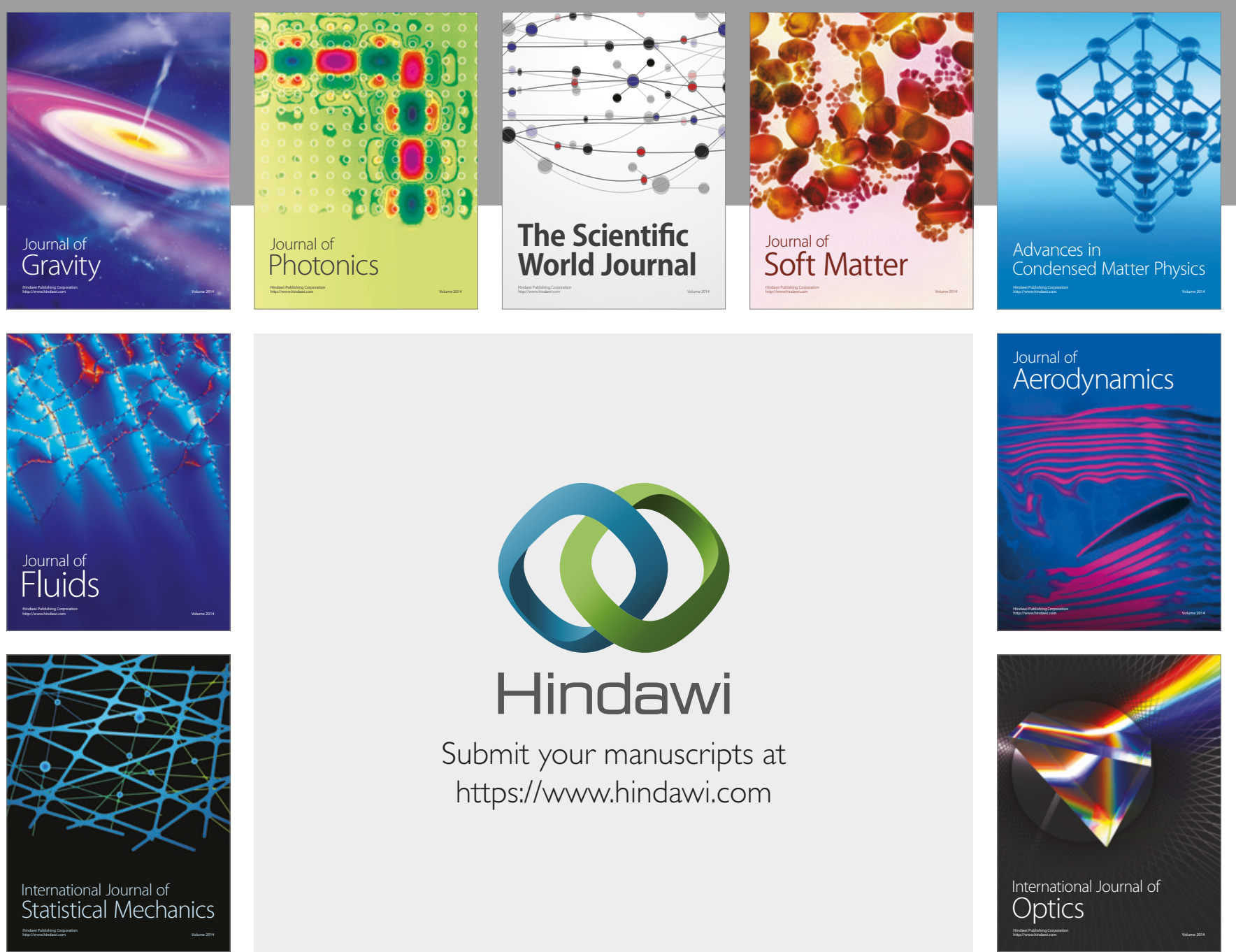

Submit your manuscripts at

https://www.hindawi.com
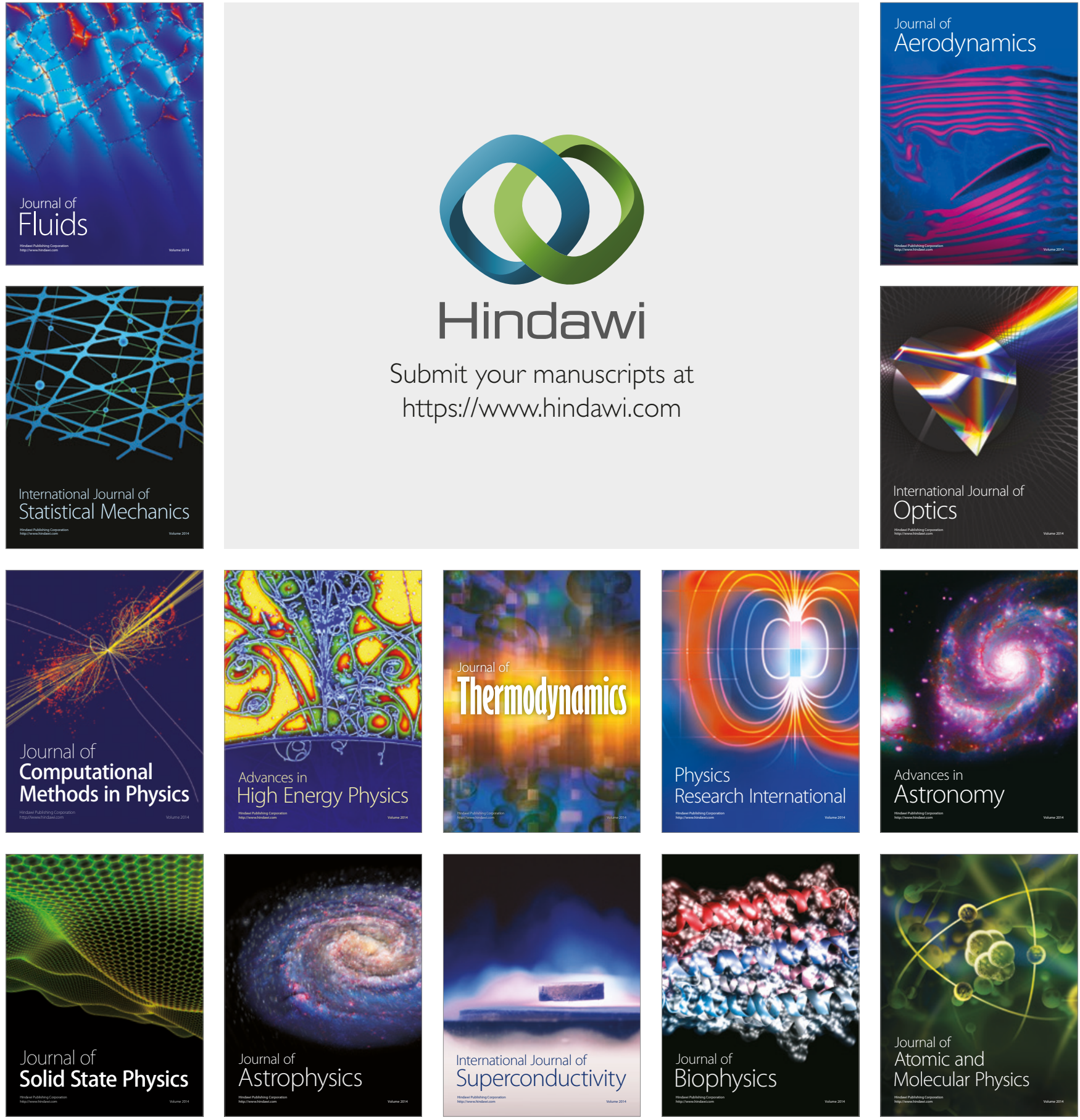